\title{
La sindrome anemica cardio-renale. Prima parte: epidemiologia e aspetti clinici
}

\author{
Rodolfo F. Rivera ${ }^{1}$, Maria Teresa Sciarrone Alibrandi ${ }^{2}$, Luca Di Lullo ${ }^{3}$, Fulvio Floccari ${ }^{4}$, Antonio De Pascalis ${ }^{5}$, Antonio Bellassi ${ }^{6}$, \\ Claudio Ronco ${ }^{7}$ \\ ${ }^{1}$ UO Nefrologia e Dialisi, Ospedale San Gerardo, ASST Monza, Monza \\ ${ }^{2}$ UO Nefrologia, Dialisi e Ipertensione, IRCCS Ospedale San Raffaele, Milano \\ ${ }^{3}$ UO Nefrologia e Dialisi, Ospedale L. Parodi- Delfino, Colleferro, Roma \\ ${ }^{4}$ UO Nefrologia e Dialisi, Ospedale L. Parodi- Delfino, Colleferro, Roma \\ ${ }^{5}$ UO Nefrologia e Dialisi, Ospedale V. Fazzi, ASL Lecce, Lecce \\ ${ }^{6}$ UO Nefrologia e Dialisi, Ospedale Sant'Anna, ASST-Lariana, Como \\ ${ }^{7}$ UO Nefrologia e Dialisi, Ospedale San Bortolo \& International Renal Research Institute Vicenza (IRRIV), Vicenza
}

The cardiorenal anemia syndrome. Part one: epidemiology and clinical aspects

Anemia is a common complication associated with congestive heart failure (CHF) and chronic kidney disease (CKD), and is often reported as a component of the cardiorenal syndrome (CRS). The triad anemia, CHF and CKD has adverse prognostic implications that have led to the reformulation of the syndrome with the term "cardiorenal anemia syndrome" (CRAS). However, there is insufficient agreement about the definition of anemia in the CHF patient, probably due to the heterogeneity of the clinical criteria and the diversity of the patient populations in different studies. The evolution of drug therapy and technology (such as resynchronization of the left ventricle) has not stopped the increase in its incidence nor the associated health system costs. Unfortunately, the current guidelines do not provide specific recommendations for the adequate management of anemia in the cardiorenal patient. The pathophysiological mechanisms at the origin of anemia in CRS are complex and numerous. On the cardiovascular side the most important mechanisms are activation of the sympathetic and renin-angiotensin-aldosterone systems, antidiuretic hormone, and hemodilution, while those associated with CKD include reduction of the endogenous production of erythropoietin, chronic microinflammation, and iron deficiency. Consequently, anemia could represent a new clinical and therapeutic biomarker in CRS. A more comprehensive, 360-degree view, which stratifies the etiological, clinical and pathophysiological aspects, could significantly contribute to improving the prognosis of CRS patients.

Keywords: Cardiorenal anemia syndrome (CRAS), Chronic kidney disease (CKD), Congestive heart failure (CHF)

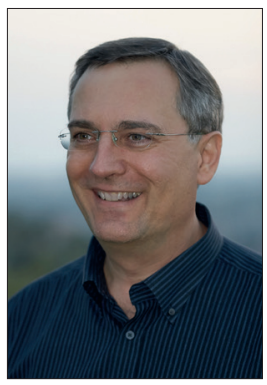

Rodolfo Rivera

\section{Introduzione}

L'interazione esistente negli aspetti funzionali tra cuore e rene è ormai nota da molto tempo. L'asse cuore-rene presenta due importanti caratteristiche, la prima di aver una natura bidirezionale che coinvolge diversi livelli di organizzazione: fisico, chimico e biologico. La seconda di svolgersi nello spazio temporale: processi acuti o cronici.

Accepted: July 31, 2017

Published online: September 12, 2017

Indirizzo per la corrispondenza:

Dr. Rodolfo F. Rivera

Divisione di Nefrologia e Dialisi

Ospedale San Gerardo, ASST Monza

Via Pergolesi, 33

20900 Monza (MB)

rodolfofrivera@gmail.com

Nonostante i diversi specialisti conoscessero molto bene questo problema dal proprio punto di vista, l'approccio multidisciplinare trova sensibili limitazioni principalmente sul piano semantico $(1,2)$. Per minimizzare tali ostacoli, si rendeva sempre più imperativa la necessità di parlare un linguaggio comune mediante l'adozione di una nuova classificazione che tenesse conto degli aspetti eziologici, fisiopatologici e clinici. La classificazione doveva inoltre distinguere l'incipit e le sequele cliniche della sindrome, differenziare l'organo primariamente coinvolto da quello secondariamente danneggiato, e infine definire la sequenza temporale dell'insorgenza del fenomeno: acuzie o cronicità.

Nel 2004, i ricercatori del National Heart, Lung, and Blood Institute (NHLBI), si sono riuniti per cercare di capire le interazioni rene-cuore, creando il Working Group on Cardio-Renal Connections in Heart Failure and Cardiovascular Disease (3). Il gruppo di studio definiva la "sindrome cardio-renale" (CRS), come la situazione nella quale la terapia per alleviare i sintomi congestivi dell'insufficienza cardiaca cronica (Chronic Heart Failure, CHF) è ulteriormente limitata dal declino della funzione renale. Tuttavia, l'assenza di una definizione chiara e la 
complessità delle interazioni cuore-rene hanno contribuito alla scarsa leggibilità per quanto riguarda la diagnosi e la gestione della CRS

Nel 2008, Ronco et al (4) definivano la CRS come la disfunzione primitiva, acuta o cronica di uno dei due organi, in grado di portare alla disfunzione secondaria, acuta o cronica dell'altro organo. La più recente definizione della CRS comprende una serie di condizioni (5), sia acute che croniche, dove l'organo di fallimento primario può essere il cuore o il rene. La CRS comprende quindi, disturbi del cuore e dei reni dove la disfunzione acuta o cronica in un solo organo è in grado di provocare la disfunzione acuta o cronica dell'altro. La definizione attuale è stata classificata in cinque sottotipi (5), la cui etimologia riflette la patologia primaria e secondaria, il tempo e la simultanea disfunzione cardiaca e renale secondaria a malattia sistemica: CRS-tipo 1, nella quale un brusco peggioramento della funzione cardiaca provoca il danno renale acuto; CRS-tipo 2, caratterizzata dall'anormalità cronica della funzione cardiaca che conduce a insufficienza renale cronica progressiva; CRS-tipo 3, nota anche come sindrome reno-cardiaca acuta, nella quale un danno renale acuto induce la disfunzione cardiaca acuta; CRS-tipo 4, o sindrome cronica reno-cardiaca, caratterizzata da insufficienza renale cronica che contribuisce al decremento della funzione cardiaca; e infine CRS-tipo 5, anche definita come CRS secondaria, caratterizzata da disfunzione cardiaca e renale concomitante dovuta a disordini sistemici acuti o cronici.

Sebbene questa classificazione esprima un ragionamento clinico e fisiopatologico lineare, non tiene conto di alcune frequenti complicanze che possono insorgere sia sul versante cardiaco che su quello renale. L'anemia è una temibile complicanza in grado di condizionare la prognosi dei nefropatici e dei cardiopatici, ed è frequentemente associata alla CRS. Questa triade sfavorevole costituita da malattia cardiaca, renale e anemia (Fig. 1) ha portato a riformulare la sindrome con la denominazione di "Sindrome anemica cardio-renale" (Cardio-Renal-Anemia-Syndrome, CRAS) (6). Numerosi studi osservazionali e diversi trial controllati e randomizzati (RCT) hanno chiaramente dimostrato l'associazione indipendente tra i bassi livelli di emoglobina $(\mathrm{Hb})$ e gli outcomes clinici avversi. Le attuali linee guida per il trattamento della CHF non prevedono raccomandazioni specifiche per la valutazione e la gestione del trattamento dell'anemia (7). Dato che la mortalità nella CHF rimane molto elevata, I'anemia potrebbe rappresentare un nuovo indicatore clinico e terapeutico nella CRS. Questa review si propone di analizzare gli aspetti epidemiologici e fisiopatologici relativi all'anemia nella CRS.

\section{La dimensione del problema}

La prevalenza della CHF $(8,9)$ e della malattia renale cronica (Chronic Kidney Disease, CKD) anche nelle fasi più avanzate (End Stage Renal Disease, ESRD) (10) stanno aumentando rapidamente e in modo sostenuto (11).

La CHF è presente in circa il $2 \%$ della popolazione generale e l'età è considerata un rilevante fattore di rischio.

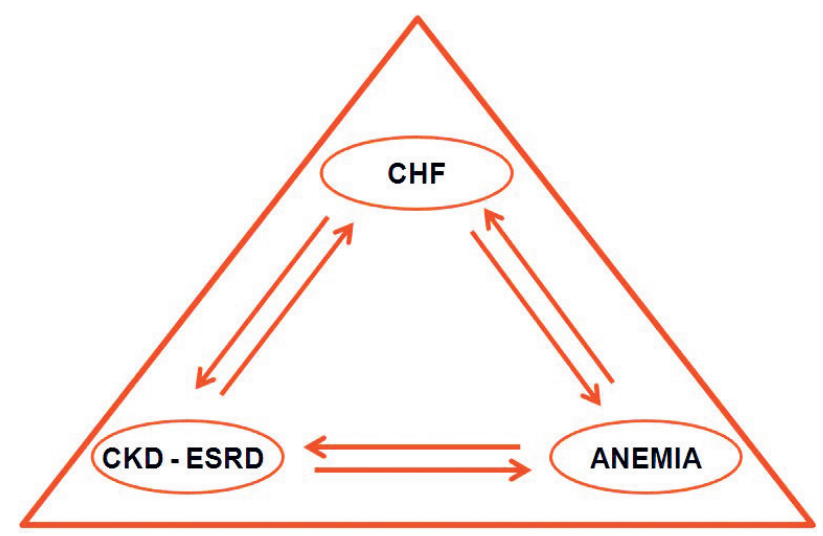

Fig. 1 - La sindrome anemica cardio-renale.

Di fatto, la prevalenza della CHF aumenta del $5 \%$ sopra i 55 anni di età e del $10 \%$ sopra gli 80 anni. La mortalità annua associata alla CHF arriva al $20 \%$ nei casi lievi e supera il $50 \%$ nei casi più severi (6). La CHF rappresenta la causa più comune di ospedalizzazione nei soggetti con più di 65 anni. Oggi negli Stati Uniti ci sono oltre 5 milioni di casi sintomatici di CHF con un incremento di 0,5 milioni ogni anno. Lo sviluppo terapeutico della CHF come conseguenza del migliore utilizzo di farmaci antagonisti dei sistemi simpatico e renina-angiotensina-aldosterone, e dell'introduzione della terapia di resincronizzazione elettrica ventricolare mediante appositi dispositivi e defibrillatori automatici, hanno migliorato sensibilmente la prognosi di questi pazienti (2). Nonostante tutto questo, la CHF continua a essere una malattia altamente letale ed estremamente costosa per i sistemi socio-sanitari $(4,5)$. Nei primi anni di questo secolo, la spesa sanitaria americana arrivava a 56 miliardi di dollari annui, e comunque i decessi annui erano circa $250.000(8,12,13)$. Il mutamento demografico che si sta verificando da qualche decennio e che definisce la nuova tipologia del paziente cronico affetto da diverse comorbidità che giungono oggi ai nostri ambulatori, contribuirà a potenziare questo fenomeno nei prossimi anni.

Circa la metà dei pazienti affetti da CHF presenta una compromissione della funzione renale espressa con l'aumento lieve dei valori di creatininemia $(\geq 1,5 \mathrm{mg} / \mathrm{dL}$ ) e/o la riduzione del filtrato glomerulare (Glomerular Filtration Rate, GFR) ( $\leq 60 \mathrm{~mL} / \mathrm{min} / \mathrm{sq})$. Entrambi i criteri sono validi per definire la CKD di media o moderata entità (14-16). È noto che la CKD si associa alla insorgenza di anemia, e che la severità dell'anemia è correlata agli stadi più avanzati di CKD proprio come conseguenza della riduzione nella produzione di eritropoietina endogena (EPO) da parte del rene. La presenza di entrambi i fattori di rischio, anemia e CKD, grava sulla progressione della CHF (14-18), configurando in questo modo un circolo vizioso noto come Sindrome anemica cardio-renale (Fig. 1). 


\section{La prevalenza dell'anemia nella CHF}

Nel 1968 l'Organizzazione Mondiale della Sanità (OMS) definisce l'anemia come una riduzione assoluta del numero complessivo dei globuli rossi circolanti (RBC) con una conseguente riduzione della concentrazione di emoglobina $(\mathrm{Hb})<13,0 \mathrm{~g} / \mathrm{dL}$ per i maschi adulti e le donne in post-menopausa e una $\mathrm{Hb}$ $<12,0 \mathrm{~g} / \mathrm{dL}$ per le donne in pre-menopausa (19). Una definizione alternativa dell'anemia associata all'insufficienza renale, stabilita nelle linee guida della National Kidney Foundation (20) prevede un valore di $\mathrm{Hb}<13,5 \mathrm{~g} / \mathrm{dL}$ nell'uomo e $<12 \mathrm{~g} / \mathrm{dL}$ nella donna adulta. L'assenza di criteri uniformi nella definizione dell'anemia associata a CHF, ha indotto i singoli studi ad adottare diversi valori soglia di $\mathrm{Hb}$, complicando sensibilmente I'interpretazione dei risultati. Allo stato attuale non c'è concordanza nella definizione di anemia (21), in particolare quando si riferisce alla CHF. La prevalenza dell'anemia riportata nei diversi RCT e negli studi di coorte varia dal $9 \%$ al 70\% (22).

Analizzando una discreta casistica di cardiopatici scompensati, Silverberg et al (15) notano che nonostante la terapia anti-scompenso utilizzando dosi massimali di ACE-inibitori, beta-bloccanti nitrati, antialdosteronici, digossina e diuretici, la refrattarietà terapeutica in termini clinici resta elevata. La presenza di anemia secondo i criteri OMS ( $\mathrm{Hb}$ $<12 \mathrm{~d} / \mathrm{dL}$ ) era del $55,6 \%$ in tutti i pazienti con CHF, e la prevalenza incrementava da 9,1\% nei pazienti NYHA classe I a $79,1 \%$ nei pazienti NYHA classe IV. Anche nello Study Anemia in a Heart Failure Population (STAMINA-HFP) che includeva una coorte di 1076 pazienti con CHF, la prevalenza di anemia viene riportata del $34 \%$ sulla base dei criteri diagnostici OMS, e solo il $12 \%$ presentava valori $\mathrm{Hb}<11 \mathrm{~g} / \mathrm{dL}$ (23). Nel registro Organized Program to Initiate Lifesaving Treatment in Patients with Heart Failure (OPTIMIZE-HF), che studiava una casistica ancora maggiore comprendente 48.612 pazienti ricoverati per un primo episodio di CHF o per una riacutizzazione, mostrava un $25 \%$ dei pazienti con $\mathrm{Hb}<10,7 \mathrm{~g} / \mathrm{dL}$ e $51,2 \%$ con $\mathrm{Hb}<12,1 \mathrm{~g} / \mathrm{dL}$ (24). Analizzando i diversi studi è possibile affermare che l'entità dell'anemia è correlata alla severità della disfunzione cardiaca. La prevalenza, inoltre, aumenta con le comorbidità (età, malnutrizione, disfunzione renale ecc.) e con le fragilità (CHF o CKD di lunga durata) (25-28).

I dati relativi all'incidenza dell'anemia nei cardiopatici scompensati sono tutt'altro che uniformi, per esempio, nel SOLVD (Studies of Left Ventricular Dysfunction) la diagnosi di anemia a un anno era del 9,6\% (29), mentre nel COMET (Carvedilol or Metoprolol European Trial) e nel Val-HeFT (Valsartan Heart Failure Trial) l'incidenza era del $14,2 \%$ e del $16,2 \%$ rispettivamente $(30,31)$. La Tabella I riporta la prevalenza dell'anemia nei principali studi riguardanti il CHF.

I motivi di tale dispersione non sono chiari. La disomogeneità delle caratteristiche cliniche, le diverse comorbidità dei pazienti arruolati e la variabilità nella definizione dei target clinici riportati nei diversi studi osservazionali sono tra i principali fattori di confondimento. Sfortunatamente non sono gli unici: è interessante notare che lo stato di volemia del paziente scompensato può rappresentare un importante confondente nello studio dell'anemia (32). La posologia della terapia diuretica e la definizione della refrattarietà terapeutica rappresentano punti di maggior divergenza interpretativa. L'emodiluizione conseguente alla ritenzione idrica e la diversità nei metodi per misurarla, rendono estremamente difficile quantificare la vera incidenza dell'anemia nel paziente cardiopatico scompensato.

\section{Effetti dell’anemia sulla CHF}

La presenza di anemia in pazienti affetti da CHF comporta una serie di alterazioni quali elevata mortalità, maggior numero di ospedalizzazione con aumento dei giorni di degenza, aggravamento della CHF caratterizzata dalla progressione della classe funzionale, secondo la classificazione della New York Heart Association (NYHA), riduzione della funzione ventricolare sistolica (LVEF) e diastolica, riduzione della capacità di esercizio fisico e della utilizzazione di ossigeno durante il picco di atti-

TABELLA I - Prevalenza dell'anemia nei principali studi con pazienti affetti da CHF

\begin{tabular}{llcc}
\hline Studio & Definizione anemia & No. Pazienti & Prevalenza \\
\hline Val-HeFT & $\mathrm{Hb}<11 \mathrm{~g} / \mathrm{dL}(\mathrm{F}),<12 \mathrm{~g} / \mathrm{dL}(\mathrm{M})$ & 5010 & $10 \%$ \\
COMET & $\mathrm{Hb}<12 \mathrm{~g} / \mathrm{dL}(\mathrm{F}),<13 \mathrm{~g} / \mathrm{dL}(\mathrm{M})$ & 3029 & $16 \%$ \\
IN-CHF & $\mathrm{Hb}<11 \mathrm{~g} / \mathrm{dL}(\mathrm{F}),<12 \mathrm{~g} / \mathrm{dL}(\mathrm{M})$ & 2411 & $16 \%$ \\
ELITE-II & $\mathrm{Hb}<12.4 \mathrm{~g} / \mathrm{dL}$ & 3044 & $17 \%$ \\
RENAISSANCE & $\mathrm{Hb}<12 \mathrm{~g} / \mathrm{dL}$ & 912 & $20 \%$ \\
PRAISE & $\mathrm{Hct}<37,6 \%$ & 1130 & $20 \%$ \\
EuroHeart Failure Survey & $\mathrm{Hb}<11 \mathrm{~g} / \mathrm{dL}$ & 9971 & $21 \%$ \\
CHARM & $\mathrm{Hb}<12 \mathrm{~g} / \mathrm{dL}(\mathrm{F}),<13 \mathrm{~g} / \mathrm{dL}(\mathrm{M})$ & 2653 & $26 \%$ \\
STAMINA-HFP & $\mathrm{Hb}<12 \mathrm{~g} / \mathrm{dL}(\mathrm{F}),<13 \mathrm{~g} / \mathrm{dL}(\mathrm{M})$ & 982 & $33 \%$ \\
OPTIME-CHF & $\mathrm{Hb}<12 \mathrm{~g} / \mathrm{dL}(\mathrm{F}),<12 \mathrm{~g} / \mathrm{dL}(\mathrm{M})$ & 906 & $49 \%$ \\
\hline
\end{tabular}

$\mathrm{F}=$ femmine; $\mathrm{M}$ = maschi; $\mathrm{Hb}=$ emoglobina; $\mathrm{Hct}=$ ematocrito.

Modificata da Tang Y-D, Katz SD (21). 


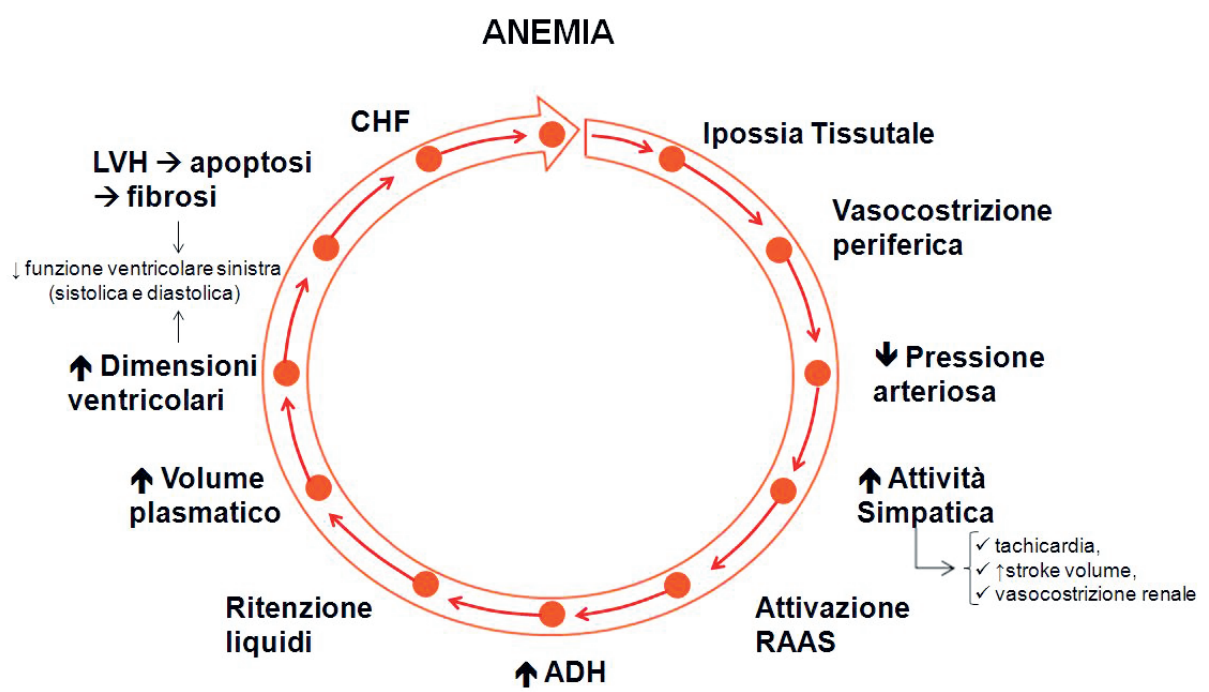

Fig. 2 - Fisiopatologia dell'anemia nelle malattie cardiovascolari. vità, riduzione della qualità di vita (QoL), aumento del Brian Natriuretic Peptide (BPN) circolante, e successiva formazione di edema periferico.

L'anemia inoltre è spesso associata a disfunzione renale che comporta una maggior progressione verso la CKD, comparsa di segni di denutrizione e riduzione del Body Mass Index (BMI), associata a riduzione dell'albumina plasmatica, delle proteine totali e del colesterolo totale. L'anemia e la malnutrizione sono associate alla risposta infiammatoria nella $\mathrm{CHF}$, dove esiste un incremento nella produzione di Tumor Necrosis Factor alpha (TNF- $\alpha$ ) e Interleukin 6 (IL6).

In molti studi di associazione è stato possibile stabilire che la presenza di anemia era associata a una classe funzionale NYHA più severa (14), e a una maggior mortalità (31). Nella maggioranza di questi studi, l'associazione anemia e CHF restava statisticamente significativa anche dopo correzione con altri fattori di rischio come la CKD e l'età mediante l'uso di analisi multivariata. L'anemia quindi, veniva considerata come un fattore di rischio indipendente per la mortalità cardiovascolare. In altri studi invece, l'anemia e la CKD hanno evidenziato un effetto additivo sull'incremento della mortalità e il rischio di progressione verso ESRD (33). I pazienti affetti da CKD con anemia raddoppiano il rischio di sviluppare $\mathrm{CHF}$ in un periodo di 10,5 anni di follow-up rispetto ai CKD non anemici (34). Nello stesso modo, i pazienti con CKD anemici aumentano l'incidenza in complicanze cardiovascolari e stroke $(35,36)$.

Dai registri internazionali emerge che solo una minima parte dei pazienti affetti da CHF (circa il 10\%) presenta una funzione renale normale, mentre circa il 50\% di loro ha una disfunzione renale rilevante (classe $K D O Q I \geq I I I)$, definita con un filtrato glomerulare $<60 \mathrm{~mL} / \mathrm{min} / 1,73 \mathrm{~m}^{2}$ (28, 37-39). La coesistenza tra anemia e disfunzione renale è più che casuale e nei pazienti con $\mathrm{CHF}$, quando ciò si verifica, ha una notevole rilevanza di carattere prognostica già a livelli fun- zionali ben lontani dall'uremia (filtrato glomerulare $<45 \mathrm{~mL}$ / $\min / 1,73 \mathrm{~m}^{2}$ ) (28).

La triade CKD, CHF e anemia (Fig. 1) è associata ad almeno 4 diversi meccanismi fisiopatogenici sottostanti che agiscono a livello tissutale: nell'aumento dell'attività simpatica, nell'attività RAAS, nello stress ossidativo e nell'attività pro-infiammatoria (40). Esistono tuttavia altri meccanismi presenti in grado di indurre alterazioni cardiache nei pazienti con CKD e anemia. L'aterosclerosi è un processo che si osserva spesso in questi pazienti. Le calcificazioni vascolari sono quattro volte più frequenti in pazienti uremici rispetto ai controlli, e le placche di ateroma crescono più velocemente $(41,42)$. L'angiogenesi eccessiva nella tunica interstiziale della parete arteriosa può condurre alla formazione di ematoma e conseguente rottura della placca fibro-calcifica. A livello endoteliale, la presenza di anemia accentua il danno microvascolare, che appare direttamente proporzionale all'ipertrofia miocardica. La difficoltà nel percorso tissutale dell'ossigeno per raggiungere la cellula miocardica aumenta a causa dei micro-ostacoli a livello del lume capillare (43).

Questi meccanismi sono alla base della strategia terapeutica da usare per contrastare l'anemia nella CRS.

\section{Eziologia dell'anemia nella CHF}

La causa più comune di anemia nella CHF è associata alla presenza di CKD, che determina una vasocostrizione renale (44), dovuta alla fibrosi interstiziale che porta a ischemia e successiva riduzione della produzione di EPO endogena (Fig. 2). La vasocostrizione periferica inoltre, causa la riduzione dei valori di pressione arteriosa, attivando il Sistema Simpatico (SS) che causa tachicardia, aumento dello stroke volume e ulteriore vasocostrizione renale. La riduzione del flusso renale attiva il sistema renina-angiotensina-aldosterone (RAAS) che incrementa la ritenzione idrosalina e stimola la produzione di ormone 
antidiuretico (ADH), causando ulteriore vasocostrizione renale. Questi meccanismi portano alla formazione di edemi periferici e aumento del volume plasmatico circolante, che inducono una riduzione della funzione ventricolare (sistolica e diastolica) aumentando lo stress di parete e l'attività cardiaca. La cellula miocardica esposta così a tale stress inizia un processo di ipertrofia al quale segue la morte cellulare per necrosi e/o apoptosi (45, 46). Una delle conseguenze più rilevante di questo fenomeno è l'aumento progressivo del diametro del ventricolo sinistro e dello spessore di parete, espresso clinicamente come ipertrofia del ventricolo sinistro (Left Ventricular Hypertrophy, LVH) eccentrica e concentrica rispettivamente (47). In questo processo fisiopatologico, la presenza di anemia può peggiorare lo scompenso cardiocircolatorio.

Tuttavia esistono altre pathways nelle quali l'anemia può essere un fattore causale di CHF. La mancanza di ossigeno nel miocardio incrementa la frequenza cardiaca e lo stroke volume, potenziando l'ischemia tissutale e quindi la morte del miocardiocita. Come è noto, gli RBC contengono diverse componenti antiossidanti per cui non c'è da sorprendersi se l'anemia è associata all'incremento dello stress ossidativo, il quale è in grado di amplificare il danno vascolare e il danno a livello della cellula del miocardio $(48,49)$.

Studi su modelli sperimentali hanno dimostrato che la CHF di per sé può indurre anemia (50). La cellula miocardica danneggiata dall'ischemia cronica secreta citochine pro-infiammatorie come il TNF- $\alpha$ (51), il quale può indurre anemia in tre modi diversi (52): mediante la riduzione di EPO endogena dai reni, interferendo con l'attività dell'EPO a livello del midollo, e inibendo il rilascio di ferro ( $\mathrm{Fe}$ ) da parte del sistema reticolo-endoteliale impedendo in questo modo la produzione di $\mathrm{Hb}$ da parte del midollo. Bolger et al (53) hanno documentato che elevati livelli di TNF- $\alpha$ e IL6 in pazienti affetti da $\mathrm{CHF}$, si associano a bassi valori di $\mathrm{Hb}$.

È interessante notare che molti pazienti affetti da CHF assumono aspirina (ASA) come parte della loro terapia, oppure sono in terapia anticoagulante orale (TAO) con warfarin. Nel corso di terapia antiaggregante o di TAO è possibile rilevare micro-perdite ematiche dal gastroenterico caratterizzate prima dalla riduzione della sideremia, che successivamente si traduce in un calo dell'Hb. La perdita cronica di Fe può portare alla riduzione progressiva dei depositi marziali (54), contribuendo sensibilmente alla genesi dell'anemia. La prevalenza della carenza marziale (Iron Deficiency Anemia, IDA) nei pazienti affetti da CHF è stata stimata nel range dal $5 \%$ al $21 \%$. Sebbene, in uno studio osservazionale (55) fino al $73 \%$ dei pazienti con CHF presentasse ridotti depositi marziali, l'anemia microcitica veniva osservata solo in una parte di questi pazienti. Comunque, un legame diretto tra CHF e IDA non è stato ancora trovato, particolarmente in assenza di certe condizioni di comorbidità in grado di ridurre l'assorbimento del $\mathrm{Fe}$, come la disfunzione ventricolare destra che determina la formazione di edema e congestione dell'apparato gastroenterico, oppure la presenza di ESRD associata a gastrite uremica. Alcune evi- denze suggeriscono che la sideropenia subclinica può compromettere la capacità aerobica indipendente dall'anemia.

L'uso di ACE-inibitori o di sartani nei pazienti con CHF è stato proposto come uno tra i fattori capaci di indurre anemia attraverso l'inibizione della produzione endogena di EPO (56).

Parte dell'anemia rilevata nei pazienti con CHF potrebbe essere dovuta agli effetti dell'emodiluizione (57). In questi pazienti è sempre difficile attribuire con una stima precisa qual è la quota di anemia relativa all'incremento del volume plasmatico circolante dovuto allo scompenso congestizio rispetto a quella dovuta alla riduzione effettiva delle RBC.

\section{Conclusioni}

L'anemia rappresenta una importante comorbilità presente nella CRS. Studi clinici osservazionali e RCT hanno documentato l'associazione indipendente tra bassi valori di $\mathrm{Hb}$ e outcomes sfavorevoli, ipotizzando come la triade anemia, CKD e CHF abbia un effetto negativo sia sulla mortalità che sulla morbilità. Almeno la metà dei pazienti con CHF presenta anche una compromissione della funzione renale, ed è noto che la severità dell'anemia è correlata agli stadi più avanzati di CKD.

La scarsa concordanza nei termini per definire l'anemia nel paziente cardiopatico ha creato una grande disomogeneità nei dati epidemiologici con rilevanti problemi interpretativi. Inoltre, l'ottimizzazione della terapia farmacologia e tecnologica, come l'introduzione dei "device" per la terapia di resincronizzazione del ventricolo sinistro non hanno arrestato la crescita della malattia né la spesa sanitaria. D'altra parte, le raccomandazioni dalle linee guida sembrano poco specifiche per gestire adeguatamente l'anemia nei pazienti affetti da CHF, particolarmente se condividono altre comorbidità come la CKD.

Per quanto riportato, appare chiaro che l'anemia potrebbe rappresentare un nuovo biomarker clinico e terapeutico nella CRS.

I meccanismi fisiopatologici alla base della genesi dell'anemia nella CRS sono complessi e numerosi. Esistono quelli associati al versante cardiovascolare, come l'attivazione del SS, RAAS, ADH, emodiluizione ecc., e quelli tipici della CKD, tra cui la riduzione della produzione endogena di EPO, la microinfiammazione cronica, la sideropenia eccetera.

Una visione più completa, "a 360 gradi", capace di identificare le diverse comorbidità del paziente (come anemia, grado di CKD, classe funzionale NYHA, e altre malattie croniche), potrebbe contribuire all'inquadramento clinico migliorando la classificazione dei pazienti affetti da CRS.

\section{Disclosures}

Financial support: No financial support was received for this submission.

Conflict of interest: The authors have no conflict of interest. 


\section{Bibliografia}

1. Di Lullo L, Floccari F, De Pascalis A, et al. Coronary artery disease (CAD) in chronic kidney disease patients. G Tec Nefrol Dial. 2016;28(1):44-52.

2. Floccari F, Di Lullo L, Rivera R, Malaguti M, Granata A, Timio M. Prevenire la morte improvvisa nel paziente uremico: mission impossible per cardionefrologi. G Tec Nefrol Dial. 2012;23(4):69-74.

3. Williams RG, Pearson GD, Barst RJ, et al; National Heart, Lung, and Blood Institute Working Group on research in adult congenital heart disease. Report of the National Heart, Lung, and Blood Institute Working Group on research in adult congenital heart disease. J Am Coll Cardiol. 2006;47(4):701-7.

4. Ronco C, Haapio M, House AA, Anavekar N, Bellomo R. Cardiorenal syndrome. J Am Coll Cardiol. 2008;52(19):1527-39.

5. Ronco C, McCullough P, Anker SD, et al; Acute Dialysis Quality Initiative (ADQI) consensus group. Cardio-renal syndromes: report from the consensus conference of the acute dialysis quality initiative. Eur Heart J. 2010;31(6):703-11.

6. Kazory A, Ross EA. Anemia: the point of convergence or divergence for kidney disease and heart failure? J Am Coll Cardiol. 2009;53(8):639-47.

7. Hunt SA, Abraham WT, Chin MH, et al. 2009 Focused update incorporated into the ACC/AHA 2005 Guidelines for the Diagnosis and Management of Heart Failure in Adults: a report of the American College of Cardiology Foundation/American Heart Association Task Force on Practice Guidelines: developed in collaboration with the International Society for Heart and Lung Transplantation. Circulation. 2009;119(14):e391-479.

8. Miller LW, Missov ED. Epidemiology of heart failure. Cardiol Clin. 2001;19(4):547-55.

9. Petrie MC, Berry C, Stewart S, McMurray JJ. Failing ageing hearts. Eur Heart J. 2001;22(21):1978-90.

10. United States Renal Data System: USRDS 2011 Annual Data Report. National Institutes of Health, National Institute of Diabetes and Digestive and Kidney Diseases, Bethesda, MD. 2011.

11. Rivera R, Floccari F, Di Lullo L, et al. La malattia renale cronica e il trattamento dello scompenso cardiaco congestizio: il ruolo del cardionefrologo. G Tec Nefrol Dial. 2012;24(1):82-94.

12. Hunt SA, Abraham WT, Chin MH, et al. 2009 focused update incorporated into the ACC/AHA 2005 Guidelines for the Diagnosis and Management of Heart Failure in Adults: a report of the American College of Cardiology Foundation/American Heart Association Task Force on Practice Guidelines: developed in collaboration with the International Society for Heart and Lung Transplantation. Circulation. 2009;119(14):e391-479.

13. O'Connell JB. The economic burden of heart failure. Clin Cardiol. 2000;23(3 Suppl):III6-10.

14. Silverberg DS, Wexler D, laina A. The role of anemia in the progression of congestive heart failure. Is there a place for erythropoietin and intravenous iron? J Nephrol. 2004;17(6):749-61.

15. Silverberg DS, Wexler D, Blum M, et al. The use of subcutaneous erythropoietin and intravenous iron for the treatment of the anemia of severe, resistant congestive heart failure improves cardiac and renal function and functional cardiac class, and markedly reduces hospitalizations. J Am Coll Cardiol. 2000;35(7):1737-44.

16. Silverberg DS, Wexler $D$, Blum $M$, laina $A$. The cardio renal anemia syndrome: correcting anemia in patients with resistant congestive heart failure can improve both cardiac and renal function and reduce hospitalizations. Clin Nephrol. 2003;60 (Suppl 1):S93-102.

17. Silverberg DS, Wexler D, Blum $M$, et al. The interaction between heart failure, renal failure and anemia - the cardiorenal anemia syndrome. Blood Purif. 2004;22(3):277-84.

18. Wexler D, Silverberg D, Sheps D, et al. Prevalence of anemia in patients admitted to hospital with a primary diagnosis of congestive heart failure. Int J Cardiol. 2004;96(1):79-87.

19. Nutritional anaemias. Report of a WHO scientific group. World Health Organ Tech Rep Ser. 1968;405:5-37.

20. NKF-DOQI clinical practice guidelines for the treatment of anemia of chronic renal failure. National Kidney FoundationDialysis Outcomes Quality Initiative. Am J Kidney Dis. off J Natl Kidney Found. 1997;30(Suppl 3):S192-240.

21. Tang YD, Katz SD. Anemia in chronic heart failure: prevalence, etiology, clinical correlates, and treatment options. Circulation. 2006;113(20):2454-61.

22. Lindenfeld J. Prevalence of anemia and effects on mortality in patients with heart failure. Am Heart J. 2005;149(3):391-401.

23. Adams KF Jr, Patterson JH, Oren RM, et al. Prospective assessment of the occurrence of anemia in patients with heart failure: results from the Study of Anemia in a Heart Failure Population (STAMINA-HFP) Registry. Am Heart J. 2009;157(5):926-32.

24. Young JB, Abraham WT, Albert NM, et al; OPTIMIZE-HF Investigators and Coordinators. Relation of low hemoglobin and anemia to morbidity and mortality in patients hospitalized with heart failure (insight from the OPTIMIZE-HF registry). Am J Cardiol. 2008;101(2):223-30.

25. Al-Ahmad A, Rand WM, Manjunath G, et al. Reduced kidney function and anemia as risk factors for mortality in patients with left ventricular dysfunction. J Am Coll Cardiol. 2001;38(4):955-62.

26. Zakai NA, Katz R, Hirsch C, et al. A prospective study of anemia status, hemoglobin concentration, and mortality in an elderly cohort: the Cardiovascular Health Study. Arch Intern Med. 2005;24;165(19):2214-20.

27. Riva E, Tettamanti M, Mosconi P, et al. Association of mild anemia with hospitalization and mortality in the elderly: the Health and Anemia population-based study. Haematologica. 2009;94(1):22-8.

28. Go AS, Yang J, Ackerson LM, et al. Hemoglobin level, chronic kidney disease, and the risks of death and hospitalization in adults with chronic heart failure: the Anemia in Chronic Heart Failure: Outcomes and Resource Utilization (ANCHOR) Study. Circulation. 2006;113(23):2713-23.

29. Ishani A, Weinhandl E, Zhao Z, et al. Angiotensin-converting enzyme inhibitor as a risk factor for the development of anemia, and the impact of incident anemia on mortality in patients with left ventricular dysfunction. J Am Coll Cardiol. 2005;45(3):391-9.

30. Komajda M, Anker SD, Charlesworth A, et al. The impact of new onset anaemia on morbidity and mortality in chronic heart failure: results from COMET. Eur Heart J. 2006;27(12):1440-6.

31. Anand IS, Kuskowski MA, Rector TS, et al. Anemia and change in hemoglobin over time related to mortality and morbidity in patients with chronic heart failure: results from Val-HeFT. Circulation. 2005;112(8):1121-7.

32. Westenbrink BD, Visser FW, Voors AA, et al. Anaemia in chronic heart failure is not only related to impaired renal perfusion and blunted erythropoietin production, but to fluid retention as well. Eur Heart J. 2007;28(2):166-71.

33. Herzog CA, Muster HA, Li S, Collins AJ. Impact of congestive heart failure, chronic kidney disease, and anemia on survival in the Medicare population. J Card Fail. 2004;10(6):467-72.

34. Muntner P, He J, Astor BC, Folsom AR, Coresh J. Traditional and nontraditional risk factors predict coronary heart disease in chronic kidney disease: results from the atherosclerosis risk in communities study. J Am Soc Nephrol JASN. 2005;16(2):529-38. 
35. Jurkovitz CT, Abramson JL, Vaccarino LV, Weintraub WS, McClellan WM. Association of high serum creatinine and anemia increases the risk of coronary events: results from the prospective community-based atherosclerosis risk in communities (ARIC) study. J Am Soc Nephrol JASN. 2003;14(11):2919-25.

36. Abramson JL, Jurkovitz CT, Vaccarino V, Weintraub WS, McClellan W. Chronic kidney disease, anemia, and incident stroke in a middle-aged, community-based population: the ARIC Study. Kidney Int. 2003;64(2):610-5.

37. Heywood JT, Fonarow GC, Costanzo MR, Mathur VS, Wigneswaran JR, Wynne J; ADHERE Scientific Advisory Committee and Investigators. High prevalence of renal dysfunction and its impact on outcome in 118,465 patients hospitalized with acute decompensated heart failure: a report from the ADHERE database. J Card Fail. 2007;13(6):422-30.

38. Owan TE, Hodge DO, Herges RM, Jacobsen SJ, Roger VL, Redfield MM. Secular trends in renal dysfunction and outcomes in hospitalized heart failure patients. J Card Fail. 2006;12(4):257-62.

39. Tarantini L, Cioffi G, Gonzini L, et al; Italian Acute Heart Failure Survey. Evolution of renal function during and after an episode of cardiac decompensation: results from the Italian survey on acute heart failure. J Cardiovasc Med. 2010;11(4):234-43.

40. Bongartz LG, Cramer MJ, Doevendans PA, Joles JA, Braam B. The severe cardiorenal syndrome: "Guyton revisited." Eur Heart J. 2005;26(1):11-7.

41. Amann K, Ritz C, Adamczak M, Ritz E. Why is coronary heart disease of uraemic patients so frequent and so devastating? Nephrol Dial Transplant. 2003;18(4):631-40.

42. Tyralla K, Amann K. Morphology of the heart and arteries in renal failure. Kidney Int Suppl. 2003;(84):S80-83.

43. Rivera RF, Mircoli L, Bonforte G, et al. Dipyridamole stress echocardiography in diagnosis and prognosis of hemodialysis patients with asymptomatic coronary disease. Hemodial Int. 2011;15(4):468-76.

44. Anand IS, Chandrashekhar Y, Ferrari R, Poole-Wilson PA, Harris PC. Pathogenesis of oedema in chronic severe anaemia: studies of body water and sodium, renal function, haemodynamic variables, and plasma hormones. Br Heart J. 1993;70(4):357-62.

45. Johnson DB, Dell'Italia LJ. Cardiac hypertrophy and failure in hypertension. Curr Opin Nephrol Hypertens. 1996;5(2):186-91.

46. Katz AM. The cardiomyopathy of overload: an unnatural growth response. Eur Heart J. 1995;16 (Suppl 0):110-4.

47. Di Lullo L, Floccari F, Rivera R, et al. Left ventricul hypertrophy in patients with chronic kidney disease. G Tec Nefrol Dial. 2014 10;26(3):281-9.

48. Grune T, Sommerburg O, Siems WG. Oxidative stress in anemia. Clin Nephrol. 2000;53(1 Suppl):S18-22.

49. Siems WG, Sommerburg O, Grune T. Erythrocyte free radical and energy metabolism. Clin Nephrol. 2000;53(1 Suppl):S9-17.

50. Iversen PO, Woldbaek PR, Tønnessen T, Christensen G. Decreased hematopoiesis in bone marrow of mice with congestive heart failure. Am J Physiol Regul Integr Comp Physiol. 2002;282(1):R166-72.

51. Herrera-Garza EH, Stetson SJ, Cubillos-Garzon A, Vooletich MT, Farmer JA, Torre-Amione G. Tumor necrosis factor-alpha: a mediator of disease progression in the failing human heart. Chest. 1999;115(4):1170-4.

52. Means RT Jr. Advances in the anemia of chronic disease. Int J Hematol. 1999;70(1):7-12.

53. Bolger AP, Sharma R. Increase in anti-inflammatory cytokine levels in chronic heart failure: a measure of treatment success or failure? Circulation. 2001;30;104(18):E97.

54. Dec GW. Anemia and iron deficiency--new therapeutic targets in heart failure? N Engl J Med. 2009;361(25):2475-7.

55. Nanas JN, Matsouka C, Karageorgopoulos D, et al. Etiology of anemia in patients with advanced heart failure. J Am Coll Cardiol. 2006;48(12):2485-9.

56. Macdougall IC. The role of ACE inhibitors and angiotensin II receptor blockers in the response to epoetin. Nephrol Dial Transplant. 1999;14(8):1836-41.

57. Androne AS, Katz SD, Lund L, et al. Hemodilution is common in patients with advanced heart failure. Circulation. 2003;107(2):226-9. 